PROCEEDINGS OF THE

AMERICAN MATHEMATICAL SOCIETY

Volume 137, Number 2, February 2009, Pages 457-465

S 0002-9939(08)09492-6

Article electronically published on July 9, 2008

\title{
NEW EQUIVALENCES FOR PATTERN AVOIDING INVOLUTIONS
}

\author{
W. M. B. DUKES, VÍT JELÍNEK, TOUFIK MANSOUR, AND ASTRID REIFEGERSTE
}

(Communicated by Jim Haglund)

\begin{abstract}
We complete the Wilf classification of signed patterns of length 5 for both signed permutations and signed involutions. New general equivalences of patterns are given which prove Jaggard's conjectures concerning involutions in the symmetric group avoiding certain patterns of length 5 and 6 . In this way, we also complete the Wilf classification of $S_{5}, S_{6}$, and $S_{7}$ for involutions.
\end{abstract}

\section{INTRODUCTION}

Pattern avoidance has proved to be a useful concept in a variety of seemingly unrelated problems, including Kazhdan-Lusztig polynomials 2], singularities of Schubert varieties [3, 4, 5, 6, 7, 16, Chebyshev polynomials [19, rook polynomials for a rectangular board [18 and various sorting algorithms, sorting stacks and sortable permutations [8, 9, 10, 20, 21, 22.

In this paper, we deal with pattern avoidance in the symmetric group $S_{n}$ and the hyperoctahedral group $B_{n}$. The group $B_{n}$, which is isomorphic to the automorphism group of the $n$-dimensional hypercube, can be represented as the group of all bijections $\omega$ of the set $X=\{-n, \ldots,-1,1, \ldots, n\}$ onto itself such that $\omega(-i)=-\omega(i)$ for all $i \in X$, with composition as the group operation. However, for our purposes it is more convenient to represent the elements of $S_{n}$ as permutation matrices, and the elements of $B_{n}$ as signed permutation matrices, where a signed permutation matrix is a $0,1,-1$-matrix with exactly one nonzero entry in every row and every column. We may also write the elements of $B_{n}$ as words $\pi=\pi_{1} \pi_{2} \ldots \pi_{n}$ in which each of the letters $1,2, \ldots, n$ appears, possibly barred to signify negative letters; a matrix $p$ corresponds to the word $\pi$ such that $p_{i j}=1$ if $\pi_{i}=j, p_{i j}=-1$ if $\pi_{i}=-j$, and $p_{i j}=0$ otherwise. In our paper, we will make no explicit distinction between these two representations of a signed permutation. Let $I_{n}$ and $S I_{n}$ be the set of involutions in $S_{n}$ and $B_{n}$, respectively. Note that involutions correspond precisely to symmetric matrices.

A signed permutation $\pi \in B_{n}$ is said to contain the pattern $\tau \in B_{k}$ if there exists a sequence $1 \leq i_{1}<i_{2}<\ldots<i_{k} \leq n$ such that $\left|\pi_{i_{a}}\right|<\left|\pi_{i_{b}}\right|$ if and only if

Received by the editors November 21, 2007, and, in revised form, January 22, 2008.

2000 Mathematics Subject Classification. Primary 05A15; Secondary 05A05.

Key words and phrases. Forbidden subsequences, pattern avoiding permutations, pattern avoiding involutions, signed permutations, Wilf equivalence.

The second author was supported by project 201/05/H014 of the Czech Science Foundation and project MSM0021620838 of the Czech Ministry of Education.

(C)2008 American Mathematical Society 
$\left|\tau_{a}\right|<\left|\tau_{b}\right|$ and $\pi_{i_{a}}>0$ if and only if $\tau_{a}>0$ for all $1 \leq a, b \leq k$. Otherwise, $\pi$ is called a $\tau$-avoiding permutation. Note that $\pi$ contains $\tau$ if and only if the matrix representing $\pi$ contains the matrix representing $\tau$ as a submatrix. By $M(\tau)$ we denote the set of all elements of $M$ which avoid the pattern $\tau$.

Two signed patterns $\sigma$ and $\tau$ are called Wilf equivalent, in symbols $\sigma \sim \tau$, if they are avoided by the same number of signed $n$-permutations, i.e., if $\left|B_{n}(\sigma)\right|=\left|B_{n}(\tau)\right|$ for each $n \geq 1$. Similarly, $\sigma$ and $\tau$ are called $I$-Wilf equivalent, denoted by $\sigma \stackrel{I}{\sim} \tau$, if $\left|S I_{n}(\sigma)\right|=\left|S I_{n}(\tau)\right|$ for each $n$. Note that two unsigned permutations $\sigma, \tau \in S_{k}$ are Wilf-equivalent if and only if they satisfy the identity $\left|S_{n}(\sigma)\right|=\left|S_{n}(\tau)\right|$ for each $n$, and they are I-Wilf equivalent if and only if they satisfy $\left|I_{n}(\sigma)\right|=\left|I_{n}(\tau)\right|$ for each $n$. The classification given by the Wilf equivalence is slightly coarser than that which is based on the symmetries of permutations, that is, the mappings generated by the reversal, transpose, and barring operation. The same is true for the I-Wilf equivalence, where the available symmetries are generated by the two diagonal reflections and the barring operation.

The question of whether two patterns are Wilf equivalent or not is difficult to answer in many cases. By the few generic equivalences known so far, it has been possible to completely determine the Wilf classes of $S_{n}$ up to level $n=7$. The decomposition of $S_{n}$ into I-Wilf classes has been completely determined for $n=4$ and almost solved for $n=5$ as well. Jaggard 14 conjectured the last case of a possible equivalence for patterns of length 5: 12345 (or equivalently, 54321) and 45312 are equally restrictive for $I_{n}$ up to $n=11$.

Continuing the I-Wilf classification of signed patterns that began in 13, we will first prove a general equivalence result which confirms Jaggard's conjecture mentioned above, as well as another conjecture he made about the equivalence of certain patterns of length 6 . The correspondence behind this result is based on a bijection between pattern avoiding transversals of Young diagrams given by Backelin, West and Xin [1. In this way, we complete the classification of $S_{5}$ with respect to $\stackrel{I}{\sim}$, which is fundamental for the analogous classification of $B_{5}$. The result even covers all missing I-Wilf equivalences in $S_{6}$ and $S_{7}$.

Furthermore, we will show that barring some blocks of a signed block diagonal pattern preserves the Wilf class of the pattern, and it also (under some additional assumptions) preserves the I-Wilf class. These results not only allow us to determine the Wilf as well as the I-Wilf classes in $B_{5}$, but they also have consequences for longer signed patterns.

\section{JAGGARD'S CONJECTURES}

In 2003, Jaggard 14 proved the equivalences $12 \tau \stackrel{I}{\sim} 21 \tau$ and $123 \tau \stackrel{I}{\sim} 321 \tau$, and completed the classification of $S_{4}$ according to pattern avoidance by involutions in this way. Furthermore, he conjectured that

(1) $12 \ldots k \tau \stackrel{I}{\sim} k(k-1) \ldots 1 \tau$ for any $k \geq 1$,

(2) $12345 \stackrel{I}{\sim} 45312$ (or equivalently, $54321 \stackrel{I}{\sim} 45312$ ),

(3) $123456 \stackrel{I}{\sim} 456123 \stackrel{I}{\sim} 564312$ (or equivalently, $654321 \stackrel{I}{\sim} 456123$ ).

In [1], Backelin, West and Xin defined a transformation to prove $12 \ldots k \tau \sim$ $k(k-1) \ldots 1 \tau$. (As already mentioned in 13, their proof also works for a signed pattern $\tau$.) This map acts not only on permutation matrices, but more generally on transversals of Young diagrams. Bousquet-Mélou and Steingrímsson [11] showed 
that this map commutes with the diagonal reflection of the diagram, which proves the first of the three conjectures above. From this result, it follows that

$$
\left(\begin{array}{ccc}
\alpha_{k} & 0 & 0 \\
0 & \chi & 0 \\
0 & 0 & \alpha_{l}
\end{array}\right) \stackrel{I}{\sim}\left(\begin{array}{ccc}
\beta_{k} & 0 & 0 \\
0 & \chi & 0 \\
0 & 0 & \beta_{l}
\end{array}\right)
$$

for every signed permutation matrix $\chi$ and any $k, l \geq 0$, where $\alpha_{n}$ and $\beta_{n}$ denote the $n \times n$ diagonal and antidiagonal permutation matrices corresponding to $12 \ldots n$ and $n(n-1) \ldots 1$, respectively. In this section, we will show that

$$
\left(\begin{array}{cccc}
0 & 0 & 0 & \alpha_{k} \\
0 & 0 & \chi & 0 \\
0 & \chi^{t} & 0 & 0 \\
\alpha_{k} & 0 & 0 & 0
\end{array}\right) \stackrel{I}{\sim}\left(\begin{array}{cccc}
0 & 0 & 0 & \beta_{k} \\
0 & 0 & \chi & 0 \\
0 & \chi^{t} & 0 & 0 \\
\beta_{k} & 0 & 0 & 0
\end{array}\right) \quad \text { and } \quad\left(\begin{array}{ccccc}
0 & 0 & 0 & 0 & \alpha_{k} \\
0 & 0 & 0 & \chi & 0 \\
0 & 0 & 1 & 0 & 0 \\
0 & \chi^{t} & 0 & 0 & 0 \\
\alpha_{k} & 0 & 0 & 0 & 0
\end{array}\right) \stackrel{I}{\sim}\left(\begin{array}{ccccc}
0 & 0 & 0 & 0 & \beta_{k} \\
0 & 0 & 0 & \chi & 0 \\
0 & 0 & 1 & 0 & 0 \\
0 & \chi^{t} & 0 & 0 & 0 \\
\beta_{k} & 0 & 0 & 0 & 0
\end{array}\right)
$$

where $\chi^{t}$ denotes the transpose of $\chi$. Note that, different from the general case, the reverse operation is not a symmetry for involutions, so these equivalences are really new.

Our proof will also use the Backelin, West and Xin bijection [1. Therefore, let us first recall the extended notion of pattern avoidance they have used. A Young diagram (or Young shape) is a top-justified and left-justified array of cells, i.e., an array whose rows have nonincreasing lengths from top to bottom, and its columns have nonincreasing lengths from left to right. A cell of a Young shape is called a corner if the array obtained by removing the cell is still a Young shape. Occasionally, it will be convenient to use top-right justified diagrams instead of the top-left justified diagrams defined above. We will refer to the top-right justified shapes as $N E$-shapes to avoid confusion with the ordinary Young shapes.

A (signed) transversal of a Young diagram $\lambda$ is an assignment of 0's and 1's (of 0 's, 1 's and -1 's) to the cells of $\lambda$ such that each row and column contains exactly one nonzero entry. A sparse filling of $\lambda$ is an arrangement of 0 's, 1 's and -1 's which has at most one nonzero entry in every row and column.

For a $k \times k$ permutation matrix $\tau$, we say that a filling $L$ of a shape $\lambda$ contains $\tau$ if there exists a $k \times k$ subshape within $\lambda$ whose induced filling is equal to $\tau$. The set of all transversals (or signed transversals) of a shape $\lambda$ which do not contain $\tau$ is denoted by $S_{\lambda}(\tau)$ (or $B_{\lambda}(\tau)$, respectively). Two signed permutation matrices $\sigma$ and $\tau$ are called shape Wilf equivalent if $\left|B_{\lambda}(\sigma)\right|=\left|B_{\lambda}(\tau)\right|$ for all Young shapes $\lambda$. Shape Wilf equivalence clearly implies Wilf equivalence. We will also say that $\sigma$ and $\tau$ are NE-shape Wilf equivalent if $\left|B_{\lambda}(\sigma)\right|=\left|B_{\lambda}(\tau)\right|$ for each NE-shape $\lambda$. Observe that if $\sigma$ and $\tau$ are permutation matrices, then they are shape Wilf equivalent if and only if $\left|S_{\lambda}(\sigma)\right|=\left|S_{\lambda}(\tau)\right|$ for each Young diagram $\lambda$.

By [1, Proposition 2.2], $\alpha_{k}$ and $\beta_{k}$ are shape Wilf equivalent for all $k$. The following proposition, which is also largely based on [1, will allow us to extend this equivalence to more general patterns.

Proposition 2.1. Let $\lambda$ be a Young shape, and let $\chi, \chi_{1}, \chi_{2}$ be signed permutations, such that $\chi_{1}$ and $\chi_{2}$ are shape Wilf equivalent. We set

$$
\theta=\left(\begin{array}{cc}
\chi_{1} & 0 \\
0 & \chi
\end{array}\right) \text { and } \omega=\left(\begin{array}{cc}
\chi_{2} & 0 \\
0 & \chi
\end{array}\right) \text {. }
$$

There is a bijection between $\theta$-avoiding and $\omega$-avoiding sparse fillings of $\lambda$. This bijection preserves the number of nonzero entries in each row and column; in particular, $\theta$ and $\omega$ are shape Wilf equivalent. Furthermore, if $\chi$ is nonempty, the bijection preserves the values of the filling in the corners of $\lambda$. 
Proof. The proof is essentially the same as the proof given in [1, Proposition 2.3]. We briefly sketch the argument here. By assumption, there is a bijection $\varphi$ between the $\chi_{1}$-avoiding and $\chi_{2}$-avoiding signed transversals of an arbitrary Young shape. Let $L$ be an arbitrary $\theta$-avoiding sparse filling of $\lambda$. Let us colour a cell of $\lambda$ if there is no occurrence of $\chi$ to the southeast of this cell. Also, if $\lambda$ has a row or column where all the uncoloured cells contain zeros, then we colour each cell of this row or column. Note that if $\chi$ is nonempty, then all the corners of $\lambda$ are coloured. The uncoloured cells induce a $\chi_{1}$-avoiding signed transversal of a Young subdiagram of $\lambda$. We apply the bijection $\varphi$ to the subdiagram of uncoloured cells, and preserve the filling of all the coloured cells. This transforms the original filling of $\lambda$ into a $\omega$-avoiding sparse filling. This transformation is a bijection which has all the claimed properties.

Note that Proposition 2.1 yields some information even when $\chi$ is the empty matrix. In such a situation, the proposition shows that a bijection between pattern avoiding signed transversals can be extended to a bijection between patternavoiding sparse fillings by simply ignoring the rows and columns with no nonzero entries.

We will now show how the results on shape Wilf equivalence may be applied to obtain new classes of I-Wilf equivalent patterns. Let us first give the necessary definitions. For an $n \times n$ matrix $\pi$ let $\pi^{+}$denote the subfilling of $\pi$ formed by the cells of $\pi$ which are strictly above the main diagonal, and let $\pi_{0}^{+}$denote the subfilling formed by the cells on the main diagonal and above it. For example, for $\pi=2 \overline{4} 31$ we have

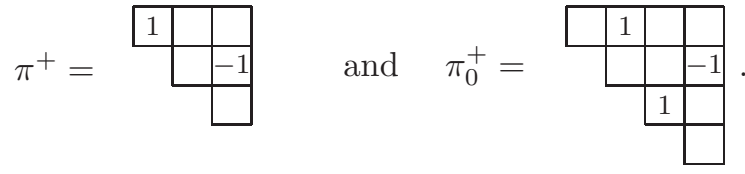

The coordinates of the entries in $\pi$ are used for the cells of $\pi^{+}$as well. Thus, for instance, the cell $(1,2)$ is the top-left corner of $\pi^{+}$. Analogously, we define $\pi^{-}$to be the filled shape corresponding to the entries strictly below the main diagonal of $\pi$. Clearly, a symmetric matrix $\pi$ is completely determined by $\pi_{0}^{+}$. Observe that a symmetric $0,1,-1$-matrix $\pi$ is a signed involution if and only if, for every $i=1, \ldots, n$, the filling $\pi_{0}^{+}$has exactly one nonzero entry in the union of all cells of the $i$-th row and $i$-th column.

Note that $i$ is a fixed point of a signed involution $\pi$, that is $\left|\pi_{i}\right|=i$, if and only if the $i$-th row and the $i$-th column of $\pi^{+}$have all entries equal to zero. In general, a signed involution $\pi$ need not be completely determined by the filling $\pi^{+}$; however, if we have two signed involutions $\pi, \rho$ with $\pi^{+}=\rho^{+}$, then $\pi$ and $\rho$ only differ by the signs of their fixed points. If $\pi$ is a signed involution, then, for each $i=1, \ldots, n$, the filling $\pi^{+}$has at most one nonzero entry in the union of the $i$-th row and $i$-th column; conversely, any filling $\pi^{+}$of an appropriate shape with these properties can be extended into a signed involution $\pi$, which is determined uniquely up to the sign of its fixed points.

For a signed permutation $\sigma$, let $\sigma^{\prime}$ denote the involution $\left(\begin{array}{cc}0 & \sigma \\ \sigma^{t} & 0\end{array}\right)$, where $\sigma^{t}$ is the transpose of $\sigma$. We are now ready to state our first result on I-Wilf equivalence. 
Theorem 2.2. If $\sigma$ and $\tau$ are two NE-shape Wilf equivalent signed permutation matrices, then $\sigma^{\prime} \stackrel{I}{\sim} \tau^{\prime}$. Moreover, the bijection between $S I_{n}\left(\sigma^{\prime}\right)$ and $S I_{n}\left(\tau^{\prime}\right)$ preserves fixed points.

Proof. Let $\pi \in S I_{n}$ be an involution. We claim that $\pi$ avoids $\sigma^{\prime}$ if and only if $\pi^{+}$ avoids $\sigma$. To see this, notice that any occurrence of $\sigma^{\prime}$ in $\pi$ can be restricted either to an occurrence of $\sigma$ in $\pi^{+}$or an occurrence of $\sigma^{t}$ in $\pi^{-}$. However, since $\pi^{+}$is the transpose of $\pi^{-}$, we know that $\pi^{-}$contains $\sigma^{t}$ if and only if $\pi^{+}$contains $\sigma$. The converse is even easier to see.

Let us choose $\pi \in S I_{n}\left(\sigma^{\prime}\right)$. Since $\pi^{+}$is a sparse $\sigma$-avoiding filling, we may apply the bijection from Proposition 2.1 (adapted for NE-shapes) to $\pi^{+}$to obtain a $\tau$-avoiding sparse filling of the same shape, which has a nonzero entry in a row $i$ (or column $i$ ) whenever $\pi^{+}$has a nonzero entry in the same row (or column, respectively). Hence this filling also corresponds to an involution, more exactly, to $\rho^{+}$for an involution $\rho \in S I_{n}$. Furthermore, the fixed points of $\rho$ are in the same position as the fixed points of $\pi$, because the position of the fixed points is determined by the zero rows and columns, which are preserved by the bijection from Proposition 2.1. By defining the signs of the fixed points of $\rho$ to be the same as the signs of the fixed points of $\pi$, the involution $\rho$ is determined uniquely. Clearly, since $\rho^{+}$avoids $\tau$, we know that $\rho$ avoids $\tau^{\prime}$. Each step of this construction can be inverted, which proves the bijectivity. Furthermore, the bijection preserves fixed points by construction.

By a similar reasoning, we obtain an analogous result for patterns of odd size. For a signed permutation $\sigma$, let $\sigma^{\prime \prime}$ denote the involution matrix

$$
\left(\begin{array}{ccc}
0 & 0 & \sigma \\
0 & 1 & 0 \\
\sigma^{t} & 0 & 0
\end{array}\right)
$$

and let $\sigma^{*}$ denote the signed permutation $\left(\begin{array}{ll}0 & \sigma \\ 1 & 0\end{array}\right)$.

Theorem 2.3. If $\sigma$ and $\tau$ are $N E$-shape Wilf equivalent, then $\sigma^{\prime \prime} \stackrel{I}{\sim} \tau^{\prime \prime}$. Moreover, the bijection between $S I_{n}\left(\sigma^{\prime \prime}\right)$ and $S I_{n}\left(\tau^{\prime \prime}\right)$ preserves fixed points.

Proof. By an argument analogous to the proof of Theorem 2.2. we may observe that an involution $\pi$ avoids $\sigma^{\prime \prime}$ if and only if $\pi_{0}^{+}$avoids the pattern $\sigma^{*}$. By Proposition 2.1 (adapted for NE-shapes), the two patterns $\sigma^{*}$ and $\tau^{*}$ are NE-shape Wilf equivalent, and furthermore, the bijection realizing this equivalence preserves the corners of the shape. Note that in our situation, the corners correspond exactly to the diagonal cells of the original signed permutation matrix.

Now we consider $\pi_{0}^{+}$for an involution $\pi \in S I_{n}\left(\sigma^{\prime \prime}\right)$. By Proposition 2.1 $\pi_{0}^{+}$is in bijection with a $\tau^{*}$-avoiding filling $\rho_{0}^{+}$. Since the bijection preserves the number of nonzero entries in each row and each column of $\pi_{0}^{+}$, and it also preserves the entries on the intersection of the $i$-th row and the $i$-th column (these are precisely the corners), we know that the bijection preserves, for each $i$, the number of nonzero entries in the union of the $i$-th row and the $i$-th column. In particular, $\rho_{0}^{+}$has exactly one nonzero entry in the union of the $i$-th row and the $i$-th column, which guarantees that $\rho_{0}^{+}$can be (uniquely) extended into an involution $\rho$.

Because the bijection preserves the entries in the diagonal cells $(i, i), i=1, \ldots, n$, the permutations $\pi$ and $\rho$ have the same fixed points. This provides the required bijection. 
Let us apply these two theorems to some special cases of shape Wilf equivalent patterns. For an integer $k \geq 0$ and a signed permutation $\chi$, let us define

$$
\theta=\left(\begin{array}{cc}
0 & \alpha_{k} \\
\chi & 0
\end{array}\right) \text { and } \omega=\left(\begin{array}{cc}
0 & \beta_{k} \\
\chi & 0
\end{array}\right) .
$$

As we know, the two patterns $\theta$ and $\omega$ are NE-shape Wilf equivalent. From our results, we then obtain the following classes of I-Wilf equivalent patterns.

Corollary 2.4. We have

$$
\left(\begin{array}{cccc}
0 & 0 & 0 & \alpha_{k} \\
0 & 0 & \chi & 0 \\
0 & \chi^{t} & 0 & 0 \\
\alpha_{k} & 0 & 0 & 0
\end{array}\right) \stackrel{I}{\sim}\left(\begin{array}{cccc}
0 & 0 & 0 & \beta_{k} \\
0 & 0 & \chi & 0 \\
0 & \chi^{t} & 0 & 0 \\
\beta_{k} & 0 & 0 & 0
\end{array}\right) \quad \text { and } \quad\left(\begin{array}{ccccc}
0 & 0 & 0 & 0 & \alpha_{k} \\
0 & 0 & 0 & \chi & 0 \\
0 & 0 & 1 & 0 & 0 \\
0 & \chi^{t} & 0 & 0 & 0 \\
\alpha_{k} & 0 & 0 & 0 & 0
\end{array}\right) \stackrel{I}{\sim}\left(\begin{array}{ccccc}
0 & 0 & 0 & 0 & \beta_{k} \\
0 & 0 & 0 & \chi & 0 \\
0 & 0 & 1 & 0 & 0 \\
0 & \chi^{t} & 0 & 0 & 0 \\
\beta_{k} & 0 & 0 & 0 & 0
\end{array}\right) \text {. }
$$

The special cases $\chi=\emptyset$ and $\chi=(1)$ show both of Jaggard's conjectures to be correct.

Corollary 2.5. We have $54321 \stackrel{I}{\sim} 45312$ and $654321 \stackrel{I}{\sim} 456123 \stackrel{I}{\sim} 564312$.

\section{BARRING OF BLOCKS}

In 13 it was shown that the barring of $\tau$ in $12 \ldots k \tau$ and $k(k-1) \ldots 1 \tau$ preserves both the Wilf class and the I-Wilf class. Furthermore it was proved that

$$
\left(\begin{array}{ccc}
\alpha_{k} & 0 & 0 \\
0 & \chi & 0 \\
0 & 0 & \alpha_{k}
\end{array}\right) \stackrel{I}{\sim}\left(\begin{array}{ccc}
\alpha_{k} & 0 & 0 \\
0 & -\chi & 0 \\
0 & 0 & \alpha_{k}
\end{array}\right)
$$

for every signed permutation matrix $\chi$ and $k \geq 0$. Basically, the assertion follows from $123 \stackrel{I}{\sim} 1 \overline{2} 3$. By a similar reasoning, we can show the I-Wilf equivalence of the reversed patterns because $321 \stackrel{I}{\sim} 3 \overline{2} 1$ as well. Now we turn our attention to the general block pattern

$$
\left(\begin{array}{ccc}
\chi_{1} & 0 & 0 \\
0 & \chi_{2} & 0 \\
0 & 0 & \chi_{3}
\end{array}\right)
$$

where the $\chi_{i}$ are signed permutation matrices. First we prove the following crucial statement.

Theorem 3.1. Let $\chi_{1}$ and $\chi_{2}$ be signed permutation matrices and set

$$
\theta=\left(\begin{array}{cc}
\chi_{1} & 0 \\
0 & \chi_{2}
\end{array}\right) \text { and } \omega=\left(\begin{array}{cc}
\chi_{1} & 0 \\
0 & -\chi_{2}
\end{array}\right) \text {. }
$$

For any Young shape $\lambda$, there is a bijection between $\theta$-avoiding and $\omega$-avoiding sparse fillings of $\lambda$. The bijection preserves the position of all nonzero entries, i.e., it transforms the filling only by changing the signs of some of the entries. In particular, the patterns $\theta$ and $\omega$ are shape Wilf equivalent. Moreover, if $\lambda$ is self-conjugate and at least one of the matrices $\chi_{1}$ and $\chi_{2}$ is symmetric, then the bijection maps symmetric fillings to symmetric fillings.

Proof. Given a $\theta$-avoiding sparse filling of $\lambda$, we construct the corresponding $\omega$ avoiding filling as follows: Colour each cell of $\lambda$ for which there is an occurrence of $\chi_{1}$ to the northwest of the cell. Note that the cells left uncoloured then form a Young subdiagram of $\lambda$. By assumption, the coloured part does not contain $\chi_{2}$. Switching the signs of all entries of this part consequently yields a signed transversal of $\lambda$ which avoids $\omega$. Note that even after the transformation has been performed, it is still true that the coloured cells are precisely those cells that have an occurrence of $\chi_{1}$ to their northwest. The transformation may have created new copies of $\chi_{1}$ in 
the diagram, but it may easily be seen that these copies do not alter the colouring of the cells. This shows that the transformation is indeed a bijection.

Let $\lambda$ now be self-conjugate with a symmetric $\theta$-avoiding filling. Obviously, if $\chi_{1}$ is symmetric, then a cell is coloured if and only if its reflection (along the main diagonal) is coloured. Hence the signs of both entries must have been changed, so the resulting filling is symmetric again. If $\chi_{2}$ is symmetric but $\chi_{1}$ is not, then we slightly modify the definition of the bijection. Colour a cell if there is an occurrence of $\chi_{2}$ to the southeast. The restriction to these cells is a symmetric filling of a self-conjugate subshape which avoids $\chi_{1}$. Now change the signs of all nonzeros in uncoloured cells. The resulting filling avoids $\omega$ and is still symmetric. It is again easy to see that this provides the required symmetry-preserving bijection.

An immediate consequence of the previous theorem is the following:

Corollary 3.2. For any signed permutation matrices $\chi_{1}, \chi_{2}, \chi_{3}$, we have

$$
\left(\begin{array}{ccc}
\chi_{1} & 0 & 0 \\
0 & \chi_{2} & 0 \\
0 & 0 & \chi_{3}
\end{array}\right) \sim\left(\begin{array}{ccc}
\chi_{1} & 0 & 0 \\
0 & -\chi_{2} & 0 \\
0 & 0 & \chi_{3}
\end{array}\right)
$$

Because of the symmetry property of the bijection we can prove an analogous result for pattern avoiding involutions.

Corollary 3.3. Let $\chi_{1}, \chi_{2}, \chi_{3}$ be signed permutation matrices, at least two of which are symmetric. Then we have

$$
\left(\begin{array}{ccc}
\chi_{1} & 0 & 0 \\
0 & \chi_{2} & 0 \\
0 & 0 & \chi_{3}
\end{array}\right) \stackrel{I}{\sim}\left(\begin{array}{ccc}
\chi_{1} & 0 & 0 \\
0 & -\chi_{2} & 0 \\
0 & 0 & \chi_{3}
\end{array}\right)
$$

Proof. By Theorem 3.1 the signed pattern $\operatorname{diag}\left(\chi_{1}, \chi_{2}, \chi_{3}\right)$ is I-Wilf equivalent with the signed pattern $\operatorname{diag}\left(\chi_{1}, \chi_{2},-\chi_{3}\right)$ (note that at least one of the two matrices $\operatorname{diag}\left(\chi_{1}, \chi_{2}\right)$ and $\chi_{3}$ is symmetric). By the same argument, the pattern $\operatorname{diag}\left(\chi_{1}, \chi_{2}, \chi_{3}\right)$ is I-Wilf equivalent with $\operatorname{diag}\left(\chi_{1},-\chi_{2},-\chi_{3}\right)$. Combining these facts with the observation that changing the signs of all three blocks clearly preserves the I-Wilf class, we may even conclude that any matrix obtained by changing the signs of any of the three blocks is I-Wilf equivalent to the original matrix.

Combining Theorem 3.1 with Theorems 2.2 and 2.3, we obtain more classes of I-Wilf equivalent patterns. The following corollary gives an example.

Corollary 3.4. Let $\chi_{1}$ and $\chi_{2}$ be signed permutation matrices. Then we have

$$
\left(\begin{array}{ccccc}
0 & 0 & 0 & 0 & \chi_{1} \\
0 & 0 & 0 & \chi_{2} & 0 \\
0 & 0 & \varepsilon & 0 & 0 \\
0 & \chi_{2}^{t} & 0 & 0 & 0 \\
\chi_{1}^{t} & 0 & 0 & 0 & 0
\end{array}\right) \stackrel{I}{\sim}\left(\begin{array}{ccccc}
0 & 0 & 0 & 0 & \chi_{1} \\
0 & 0 & 0 & -\chi_{2} & 0 \\
0 & 0 & \varepsilon & 0 & 0 \\
0 & -\chi_{2}^{t} & 0 & 0 & 0 \\
\chi_{1}^{t} & 0 & 0 & 0 & 0
\end{array}\right)
$$

where $\varepsilon$ is empty or $\varepsilon=(1)$.

\section{Classification}

The proof of Jaggard's conjecture provides the complete classification of the I-Wilf equivalences among the patterns from $S_{5}$. It turns out that there are 36 different classes (in comparison with 45 symmetry classes). By the results of [13, it is known that $B_{5}$ has at most 405 I-Wilf equivalence classes. Applying the new equivalences, we obtain 402 classes which are definitively different. (By the symmetries of an involutive permutation, the patterns are divided into 566 classes.) [12, 
Table 1] shows representatives of all classes, each with the number of involutions in $S I_{9}, \ldots, S I_{12}$ avoiding the patterns of this class. The enumeration is done for $n=9$ in any case; higher levels are only computed up to the final distinction. Classes containing patterns of $S_{5}$ are in bold; hence the classification of $S_{5}$ according to the I-Wilf equivalence can be obtained from the table as well.

The classification of the patterns of $B_{5}$ by Wilf equivalence becomes complete by Corollary 3.2. The relations given in 13 did not cover seven pairs of patterns whose Wilf equivalence was indicated by numerical results. All these cases are proved now by the corollary. Consequently, $B_{5}$ falls into 130 Wilf classes (in comparison with 284 symmetry classes). See [13, Table 7] for the complete list.

The bijections of Theorem 2.2 and Theorem 2.3 also provide the complete classification of $S_{6}$ and $S_{7}$ with respect to the I-Wilf equivalence. [12, Table 2] lists all classes of $S_{6}$ obtained by all equivalences, already known (see [13] and the references therein) or proven here. As the enumeration of involutions in $I_{12}$ avoiding the patterns shows, they are different. In a similar way, we obtain 1291 Wilf classes for $S_{7}$, whose table is available from [17.

It is very possible that the results given here and in 13 suffice to solve the I-Wilf classification of signed patterns up to length 7 . However, the numerical proof that two classes are really different for a rapidly increasing number of classes is the challenge we (and computers) have to master.

Remark 4.1. After publishing this paper on the arXiv, Aaron Jaggard mentioned that he and Joseph Marincel had shown that the patterns $(k-1) k(k-2) \ldots 312$ and $k(k-1) \ldots 21$ are I-Wilf equivalent for any $k \geq 5$ by using generating tree techniques [15].

\section{REFERENCES}

[1] J. Backelin, J. West, and G. Xin, Wilf-equivalence for singleton classes, Adv. Appl. Math. 38 (2007), no. 2, 133-148. MR2290807 (2007i:05002)

[2] D.A. Beck, The combinatorics of symmetric functions and permutation enumeration of the hyperoctahedral group, Discrete Math. 163 (1997), 13-45. MR.1428556 (98f:05149)

[3] S.C. Billey, Pattern avoidance and rational smoothness of Schubert varieties, Adv. Math. 139 (1998), 141-156. MR.1652522 (99i:14058)

[4] S. Billey, W. Jockusch and R.P. Stanley, Some combinatorial properties of Schubert polynomials, J. Algebraic Combin. 2 (1993), 345-374. MR1241505 (94m:05197)

[5] S. Billey and T. Kai Lam, Vexillary elements in the hyperoctahedral group, J. Algebraic Combin. 8 (1998), 139-152. MR:1648468(2000d:05124)

[6] S. Billey and V. Lakshmibai, On the singular locus of a Schubert variety, J. Ramanujan Math. Soc. 15 (2000), no. 3, 155-223. MR1789826 (2001m:14074)

[7] S. Billey and G. Warrington, Kazhdan-Lusztig polynomials for 321-hexagon-avoiding permutations, J. Algebraic Combin. 13 (2001), no. 2, 111-136. MR1826948(2002f:05161)

[8] M. Bóna, Symmetry and unimodality in $t$-stack sortable permutations, J. Combin. Theory Ser. A 98 (2002), 201-209. MR.1897934 (2003g:05007a)

[9] M. Bóna, A survey of stack-sorting disciplines, Electron. J. Combin. 9:2 (2002), \#A1. MR2028290 (2004j:05012)

[10] M. Bousquet-Mélou, Multi-statistic enumeration of two-stack sortable permutations, Electron. J. Combin. 5 (1998), \#R21. MR1614300(99b:05001)

[11] M. Bousquet-Mélou and E. Steingrímsson, Decreasing subsequences in permutations and Wilf equivalence for involutions, J. Alg. Comb. 22 (2005), 383-409. MR2191644 (2006j:05002)

[12] Mark Dukes, Vít Jelínek, Toufik Mansour and Astrid Reifegerste, New equivalences for pattern avoiding involutions, arXiv:0708.1357, 2007.

[13] W.M.B. Dukes, T. Mansour, and A. Reifegerste, Wilf classification of three and four letter signed patterns, Discrete Math. 308:15 (2008), 3125-3133. 
[14] A.D. Jaggard, Prefix exchanging and pattern avoidance by involutions, Electronic J. Comb. 9 (2003), \#R16. MR2028285 (2005b:05008)

[15] A.D. Jaggard and J.J. Marincel, Generating tree isomorphisms for pattern-avoiding involutions, www.ams.org/amsmtgs/2098_abstracts/1023-05-1618.pdf, 2007.

[16] V. Lakshmibai and B. Sandhya, Criterion for smoothness of Schubert varieties in $S l(n) / B$, Proc. Indian Acad. Sci. Math. Sci. 100 (1990), 45-52. MR1051089(91c:14061)

[17] T. Mansour, http://www.math.haifa.ac.il/toufik/enum2005.html, 2007.

[18] T. Mansour and A. Vainshtein, Avoiding maximal parabolic subgroups of $S_{k}$, Discrete Math. Theor. Comput. Sci. 4 (2000), 67-77. MR1798705 (2001k:05016)

[19] T. Mansour and A. Vainshtein, Restricted permutations and Chebyshev polynomials, Sém. Lothar. Combin. 47 (2002), Article B47c. MR1894023 (2003a:05003)

[20] R. Tarjan, Sorting using networks of queues and stacks, J. Assoc. Comput. Mach. 19 (1972), 341-346. MR0298803 (45:7852)

[21] J. West, Permutations with forbidden subsequences and stack-sortable permutations, Ph.D. thesis, Massachusetts Institute of Technology, Cambridge (1990).

[22] J. West, Sorting twice through a stack, Theoret. Comput. Sci. 117 (1993), 303-313. MR1235186 (94j:68045)

Science Institute, University of Iceland, Reykjavík, Iceland

E-mail address: dukes@raunvis.hi.is

Department of Applied Mathematics, Charles University, Prague, Czech Republic

E-mail address: jelinek@kam.mff.cuni.cz

Department of Mathematics, University of Haifa, 31905 Haifa, Israel

E-mail address: toufik@math.haifa.ac.il

Faculty of Mathematics, University of Magdeburg, Magdeburg, Germany

E-mail address: astrid.reifegerste@ovgu.de 